\title{
An SQP-based optimisation algorithm for the derivative design of a generator
}

\author{
B. Sobhi-Najafabadi ${ }^{1}$ and Ö. Göl ${ }^{2}$ \\ ${ }^{1}$ Centre for Advanced Manufacturing Research \\ University of South Australia \\ GPO Box 2471, Adelaide 5001, Australia \\ Telephone: +61883022018 \\ Facsimile: +6183025292 \\ E-mail: Bijan.Sobhi-Najafabadi@unisa.edu.au \\ ${ }^{2}$ School of Electrical and Information Engineering \\ University of South Australia \\ GPO Box 2471, Adelaide 5001, Australia \\ Telephone: +61883023285 \\ Facsimile: +6183023384 \\ E-mail: Ozdemir.Gol@unisa.edu.au
}

\begin{abstract}
This paper briefly reviews methods of optimisation applicable to electrical machine design with emphasis on deterministic techniques, then focusing on synchronous generator design optimisation. The case selected to demonstrate the validity of the approach is that of derivative generator design, based on an existing stator lamination. A single criterion deterministic optimisation algorithm, developed using sequential quadratic programming (SQP) technique, has been employed to obtain an optimum efficiency permanent magnet synchronous generator.
\end{abstract}

\section{Key words}

Derivative design, deterministic optimisation, generator design, permanent magnet generator.

\section{Introduction}

There is an ever increasing demand for electrical machines in today's modern society for a host of different tasks ranging from household applications to astronautics. Stringent design criteria such as minimum weight and high efficiency as well as economical and environmental issues present formidable challenges for designers of such machines. Environmental issues have been very forceful in driving manufacturers for optimal design electromagnetic devices. Also, effective use of active materials is of prime importance due to economical and sustainability of natural resources.

There is a gamut of mathematical optimisation techniques applicable to design optimisation of electrical machines to ensure that the machine designed meets the ever stringent criteria for its operation.

In the following a brief overview of types of optimisation problem and methods applicable to electrical machine design will precede focussing on synchronous generator design optimisation. An example is then given to demonstrate the application of a deterministic algorithm, based on sequential quadratic programming (SQP), to the optimal design of a permanent magnet synchronous generator [1].

\section{Types of Optimisation Problem}

The range of optimisation problems is vast. Within this range, requirements are many and varied, giving rise to a vast array of optimisation techniques. Thus, it is important to categorise optimisation problems. Categorisation aids in seeking and finding a suitable way of formulating the problem and assists in the proper selection of an optimisation algorithm.

Optimisation problems can be broadly categorised as being:

- one-dimensional or multi-dimensional;

- discrete or continuous;

- dynamic or static;

- constrained or unconstrained;

- $\quad$ single-objective or multi-objective;

- linear or non-linear.

These classifications are not exclusive; e.g. a dynamic optimisation problem may be both continuous and 
constrained. The one-dimensional optimisation problem has only one variable in its objective function. On the other hand, a multi-dimensional optimisation problem has more than one variable in its objective function. Discrete optimisation problems are the ones in which parameters can only be assigned a finite set of values. In continuous optimisation problems, the parameters can assume an infinite set of values. When the variables do not change with time, the optimisation problem is termed static optimisation. And, if the parameters of an optimisation problem are functions of time, the problem is termed dynamic optimisation. The latter can be decomposed into a sequence of static subproblems in finite time intervals. Constrained optimisation problems are ones in which parameters have limits. In contrast, parameters of unconstrained optimisation do not have any limits. A singleobjective optimisation problem alludes to an optimisation task with one objective, and multiobjective optimisation problem is with more than one objective. A linear optimisation problem is a problem with a linear objective function, and equality and inequality constraints. If such are non-linear, then the problem is classified as non-linear. In the case of electrical machines all of the foregoing categories are applicable depending on the nature of the optimisation task.

\section{Optimisation Methods Suitable for Electrical Machine Design}

The object of electrical machine design optimisation is to find - singularly or collectively - the maximum efficiency, minimum weight, maximum power-toweight ratio, and so on, for an existing design or a new design. In Fig. 1, the process block includes a design algorithm, capable of an acceptably accurate performance prediction. Input can be air gap clearance, stator yoke height, tooth height and so forth. The output is expected to be an optimal design with the optimised values of input variables yielding an optimum for the objective function.

\begin{tabular}{|c|c|c|c|c|}
\hline $\begin{array}{c}\text { Input } \\
\text { (Parameters) }\end{array}$ & $\Rightarrow$ & $\begin{array}{c}\text { Process } \\
\text { (Objective function) }\end{array}$ & $\Rightarrow$ & $\begin{array}{l}\text { Output } \\
\text { (Optimal }\end{array}$ \\
\hline
\end{tabular}

Fig. 1. Process of optimisation

Algorithms are vitally important in use with digital computers. An important feature of any algorithm is its ability to converge, meaning being able to find the solution with a finite number of iterations $[1,2]$. In the case of optimisation, this means converging to the optimal solution. There is a variety of algorithms devised for use for optimisation problems, but not all of them are suitable for every problem, sometimes not even partially. Thus, a mix of algorithms may have to be used to enhance the convergence and accuracy of the solution.

In Fig. 1, there is no feedback from output to input, implying a trial-and-error approach, also known as the traditional or the non-systematic approach. This is an approach popular amongst traditional electrical machine designers and manufacturers. Here, the designer's knowledge, intuition and experience are of vital importance. Design proceeds iteratively by repeatedly changing intuitively selected input variables and assessing the design outcomes. The designer is required to gauge the sensitivity of performance characteristics to the changing of design parameters by successive design evaluations. Finding an optimal solution this way may be more a matter of luck than the outcome of a systematic search.

These observations may justify looking for an alternative approach, which provides a systematic approach to design optimisation. Conceptually, this can be achieved by simply providing a feedback link from the output to the input in Fig. 1, in which case one can speak of systematic optimisation, as illustrated in Fig. 2.

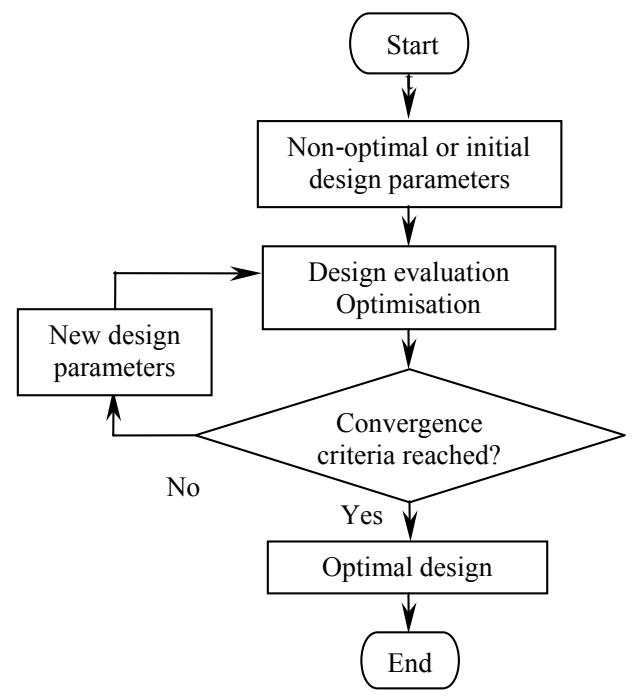

Fig. 2. Concept of systematic design optimisation

As implied in Fig. 2, the systematic optimisation starts by inputting the non-optimal design parameters to the optimisation algorithm. These parameters - or design variables - are passed onto the design algorithm, which evaluates the machine performance. The optimisation algorithm will now try to find a new set of design variables so as to yield a better performance, doing so repeatedly toward an optimal 
performance. This will continue until convergence criteria are satisfied, at which time the process will be terminated. The values returned from the last iteration just before termination provides the values, which constitute an optimal solution. Whether this point is the global optimum or not is mainly dependent on the optimisation algorithm.

The systematic approach just described removes most of the deficiencies, which are encountered in nonsystematic optimisation approaches. The most important benefit of the systematic optimisation is the elimination of the human intervention during the optimisation process.

The design algorithm, nested within the optimisation algorithm, plays a crucial role in that it must be capable of predicting the machine's performance with acceptable accuracy. In doing so a compromise is called for: a complex model representing the machine with high fidelity may be able to yield accurate results but may not be of practical use for optimisation if it places extensive computational demands on the nested algorithms. On the other hand, if the model is overly simplistic, its outputs may not be credible enough to be useful. Thus both the design algorithm and the optimisation algorithm must be selected or devised carefully with attention to speed of convergence, ability to find a "true" optimum, and credibility.

There are two main categories of optimisation algorithms: deterministic and stochastic. Deterministic algorithms, also called minimum seeking algorithms, are calculus-based [3]. One of their distinct features is their ability to move from a set of input parameters, with some deterministic sequence, and with rapid convergence to a set of output parameters. This ability occasionally turns into a handicap in that the search may be terminated prematurely at local optima, without being able to explore the rest of the feasible search for a global optimum. It is proposed that this deficiency can be overcome effectively by the use of Multilevel Coordinate Search (MCS) [4]. In contrast, stochastic algorithms - also known as exhaustive search algorithms - are probabilistic methods [5]. In these, the search is conducted with randomly selected sets of input parameters, employing some determinant sequence on a probabilistic base, leading to convergence to another set of output parameters. Stochastic search algorithms do not appear to suffer from the problem of terminating spuriously at local optima, and are capable of exploring the entire search space. Furthermore, the random nature of these methods increases the chance of finding a global optimum.

Against this backdrop, electrical machine design constitutes a constrained non-linear problem with both continuous and discrete variables, i.e. a mixedinteger programming problem.

\section{A Deterministic Optimisation Algorithm for Derivative Design}

A deterministic single-objective optimisation algorithm for the design of a PMG can be devised by means of an SQP-based optimisation algorithm [6] in conjunction with a design algorithm. The design algorithm is a systematic design and capable of complete and derivative design of permanent magnet generator $[1,7]$. The resultant design optimisation algorithm is illustrated in Fig. $3[1,8]$.

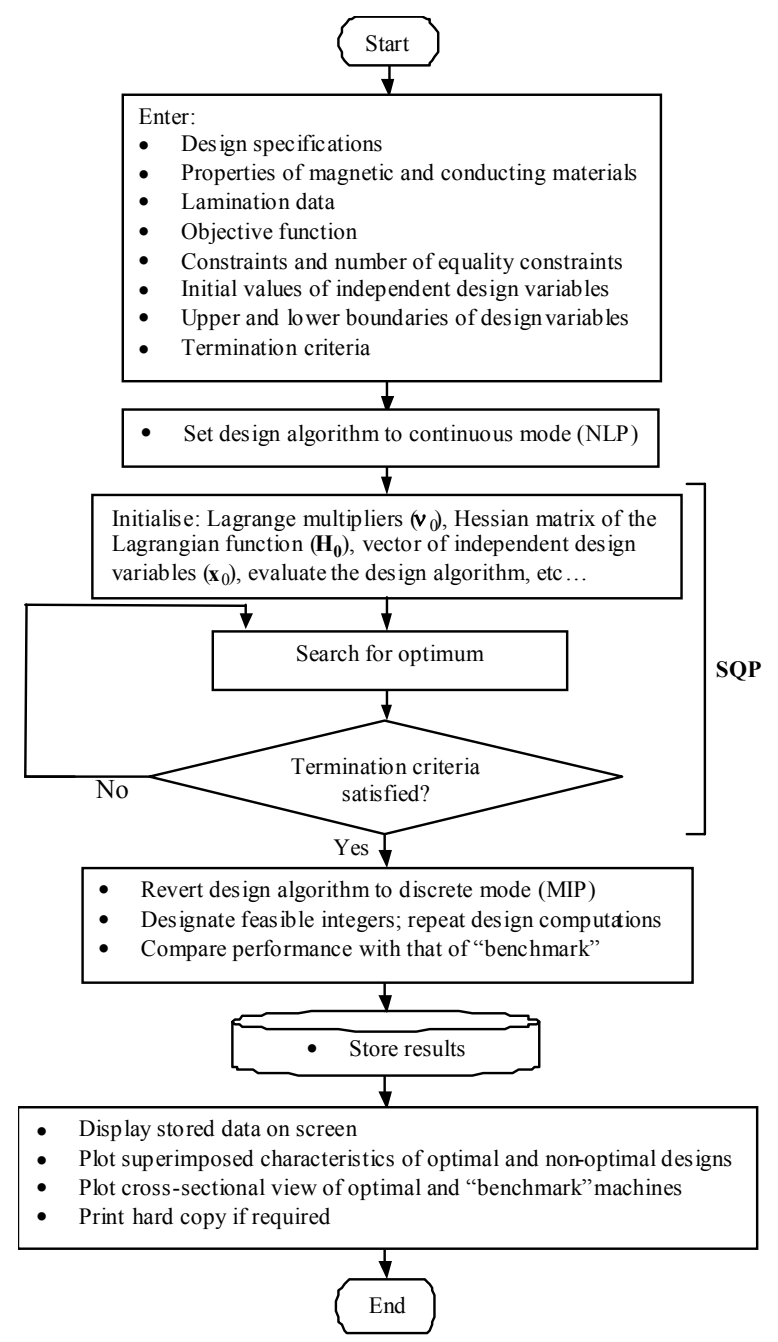

Fig. 3. Single-objective, SQP-based design optimisation algorithm 
The algorithm requires initial data including design specifications as designated by the end-user, data on magnetic properties of core and magnet materials, electrical properties of conducting materials and lamination details. It further requires nomination of a single objective from a set of objectives along with constraints, termination tolerance for the objective function, termination criterion on constraint violation and the maximum number of function evaluations. The optimisation algorithm endeavours to find a set of design variables so as to yield an optimal performance. This continues until convergence criteria are satisfied, at which time the process is terminated.

\section{Derivative Generator Design}

Design of electrical machines can proceed along two alternative paths: unconstrained and constrained. Unconstrained design is adopted when a brand new series is to be designed without any regard to existing tooling, dimensioning and manufacturing impediments. Here the designer is free to determine both the topology and winding details from the first principles, drawing these into an optimisation process if so required. In the case of constrained design, practical considerations may restrict choices. For instance, it may be stipulated that an existing lamination design be used for tooling cost reasons. In this case, the focus shifts to optimise the yield from the use of an existing core design. This constitutes derivative design optimisation.

\section{An Application Example}

The following example is to demonstrate the validity of the proposed approach to design optimisation of a permanent magnet synchronous generator. In this case an existing lamination was to be used for the stator core, with the optimisation effort focussing on rotor topology and stator winding details. Three independent design variables - namely field form factor, total electrical loading factor and air gap clearance - were manipulated. Results from two successive runs (which were identical) are given in Table I, which shows the lower and upper bounds along with the values of the three independent design variables for the optimised generator. Table II lists the constraints and their limits for execution. In Table III, the efficiency of the optimal machine is compared with that of the benchmark machine [1, 7]. Constraint violations for both the optimal machine and benchmark machine are given in Table IV, where positive numbers indicate constraint violation. Table $\mathrm{V}$ gives the performance comparison.
Fig. 4 shows the performance comparison between the benchmark generator and the optimised generator. Fig. 5 depicts the search progress. Fig. 6 compares the core geometry for the optimised and benchmark machines.

TABLE I. - Independent design variables

\begin{tabular}{|l|c|c|c|}
\hline \multirow{2}{*}{$\begin{array}{l}\text { Independent } \\
\text { design variables }\end{array}$} & \multicolumn{2}{|c|}{ Bounds } & \multirow{2}{*}{$\begin{array}{l}\text { Run } \\
1 \& 2\end{array}$} \\
\cline { 2 - 3 } & Lower & Upper & \\
\hline Field form factor & 0.65 & 0.9 & 0.9 \\
\hline $\begin{array}{l}\text { Total electrical } \\
\text { loading factor }\end{array}$ & 0.4 & 0.9 & 0.4485 \\
\hline $\begin{array}{l}\text { Air gap } \\
\text { clearance, m }\end{array}$ & 0.0009 & 0.0022 & 0.002 \\
\hline
\end{tabular}

TABLE II. - Design constraints

\begin{tabular}{|l|l|}
\hline Constraints & Limit \\
\hline Stator core length, $\mathrm{m}$ & $=$ \\
& 0.03 \\
\hline No-load stator yoke flux density, $\mathrm{T}$ & $\leq 1.7$ \\
\hline No-load stator tooth flux density, T & $\leq 1.7$ \\
\hline Temperature rise, ${ }^{\circ} \mathrm{C}$ & $\leq 45$ \\
\hline Full load voltage regulation, $\%$ & $\leq 20$ \\
\hline
\end{tabular}

TABLE III. - Comparison of efficiency

\begin{tabular}{|l|l|l|}
\hline Objective function & Benchmark & $\begin{array}{l}\text { Runs } \\
1 \& 2\end{array}$ \\
\hline Full load efficiency & 0.82802 & 0.85482 \\
\hline
\end{tabular}

TABLE IV. - Comparison of constraint violations

\begin{tabular}{|l|l|l|}
\hline Constraints & Benchmark & Runs 1\&2 \\
\hline $\mathrm{g}(1)$ & 0.005193 & 0.00041697 \\
\hline $\mathrm{g}(2)$ & -0.5361 & -0.2574 \\
\hline $\mathrm{g}(3)$ & -0.1844 & -0.2419 \\
\hline $\mathrm{g}(4)$ & -15.499 & -11.447 \\
\hline $\mathrm{g}(5)$ & -4.013 & -6.206 \\
\hline
\end{tabular}

TABLE V. - Performance comparison

\begin{tabular}{|l|l|l|l|}
\hline \multicolumn{2}{|l|}{ Performance indicator } & Runs 1\&2 & Benchmark \\
\hline \multirow{4}{*}{$\begin{array}{l}\text { Full } \\
\text { load }\end{array}$} & Current, A & 0.95628 & 0.95628 \\
\cline { 2 - 4 } & Voltage, V & 119.8432 & 121.8788 \\
\cline { 2 - 4 } & Efficiency & 0.85482 & 0.82802 \\
\cline { 2 - 4 } & $\begin{array}{l}\text { Voltage } \\
\text { regulation, } \\
\%\end{array}$ & 14.5378 & 15.987 \\
\hline $\begin{array}{l}\text { No- } \\
\text { load }\end{array}$ & Voltage, V & 140.023 & 145.0713 \\
\hline $\begin{array}{l}\text { Short } \\
\text { circuit }\end{array}$ & Current, A & 4.0605 & 2.9284 \\
\hline
\end{tabular}




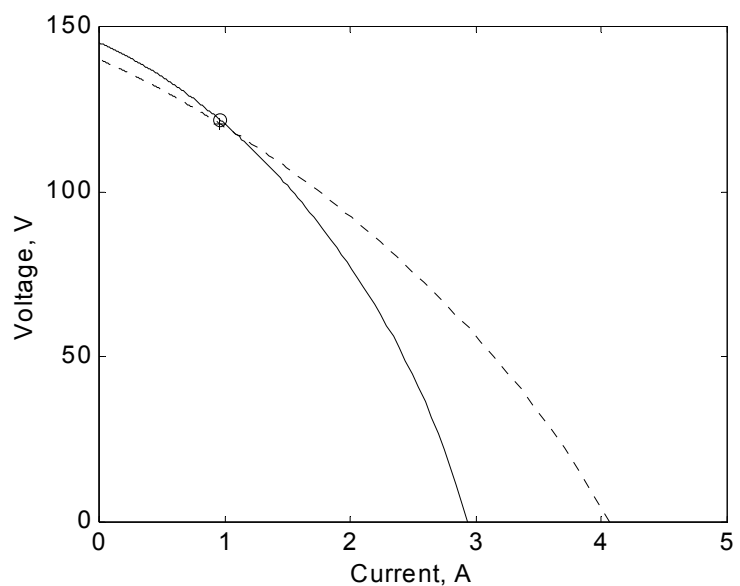

(a) Phase voltage

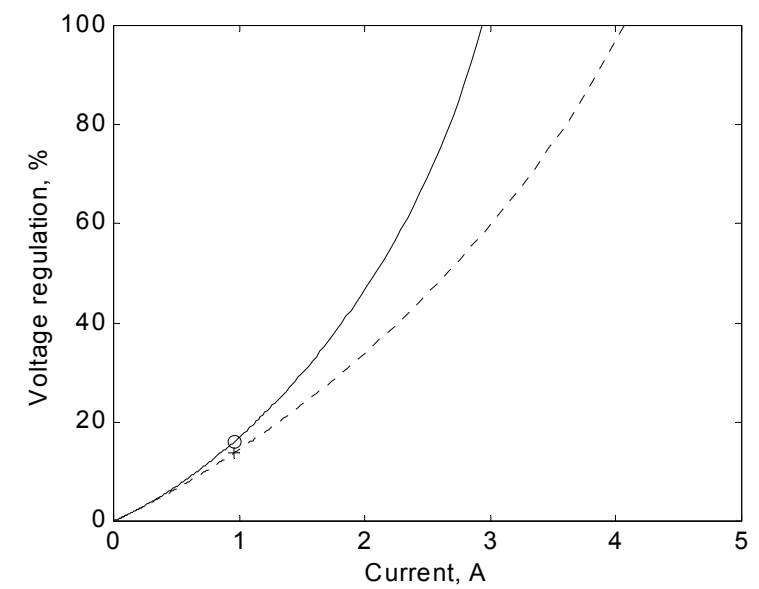

(b) Voltage regulation

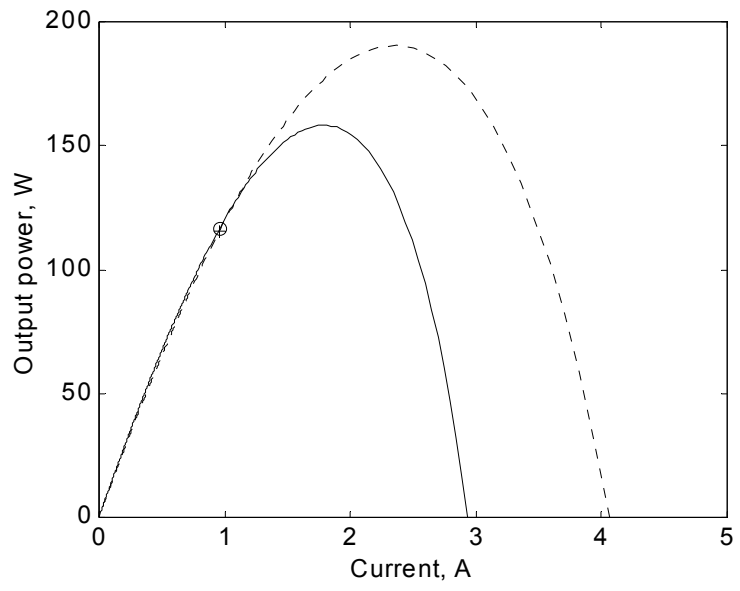

(c) Output power per phase

Fig. 4. Performance comparison for resistive load (Runs 1\&2)
---- Optimal performance Optimal at full-load

\begin{tabular}{|ccc|}
\hline f-COUNT & FUNCTION & MAX $\{\mathrm{g}\}$ \\
4 & -0.82802 & 0.016727 \\
8 & -0.83343 & 0.009583 \\
12 & -0.83647 & 0.005295 \\
16 & -0.83854 & 0.003823 \\
20 & -0.84046 & 0.000367 \\
24 & -0.84332 & $4.74 \mathrm{E}-06$ \\
28 & -0.84424 & $4.65 \mathrm{E}-06$ \\
32 & -0.84613 & $4.56 \mathrm{E}-06$ \\
36 & -0.84902 & $4.34 \mathrm{E}-06$ \\
40 & -0.85102 & $4.14 \mathrm{E}-06$ \\
44 & -0.85451 & $8.18 \mathrm{E}-08$ \\
48 & -0.85478 & $7.17 \mathrm{E}-10$ \\
49 & -0.85479 & $2.13 \mathrm{E}-10$ \\
Optimisation Converged Successfully \\
\multicolumn{3}{|}{ Active Constraints: } \\
4 & \multicolumn{3}{c}{} \\
4
\end{tabular}

Fig. 5. Search progress reported during Run 1

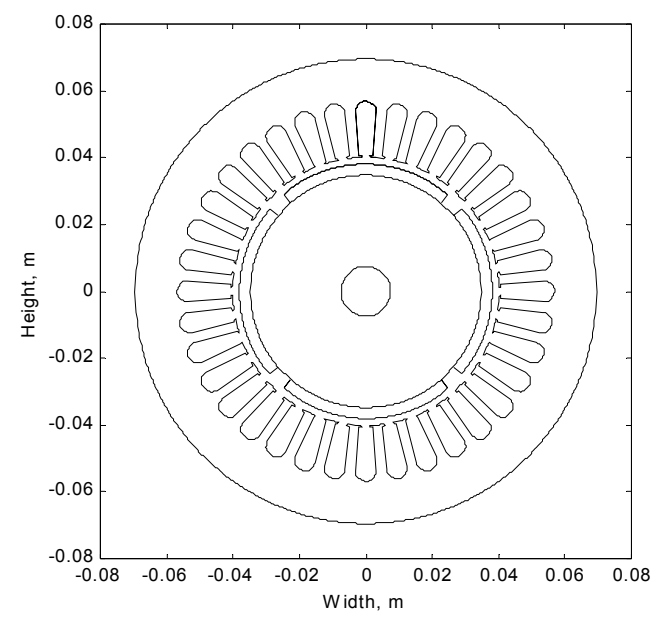

(a) Optimal generator

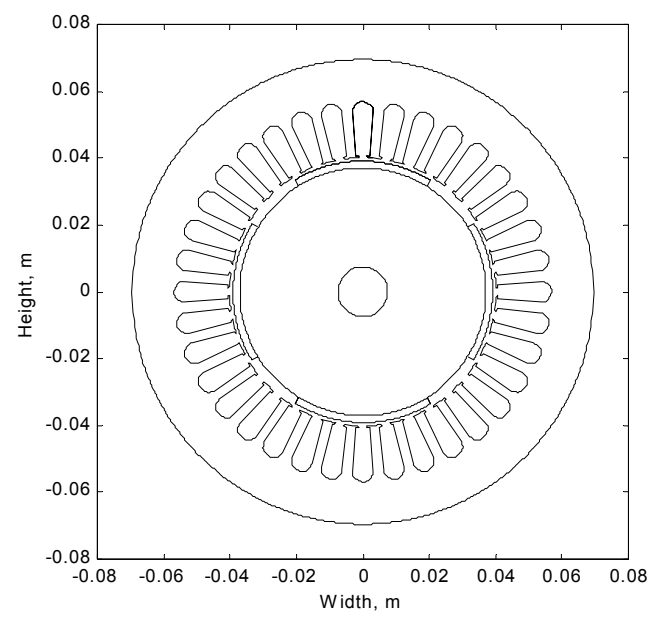

(b) Benchmark generator

Fig. 6. Core geometry (Runs 1\&2) 


\section{Conclusion}

Deterministic algorithms have greater convergence accuracy than do the stochastic ones. They are also more robust than their counterparts using stochastic approaches such as evolutionary computation.

The example given demonstrates the use, effectiveness and suitability of a practical optimisation algorithm, based on deterministic methods, to the derivative design of a permanent magnet generator. Although the emphasis has been on derivative design, the algorithm discussed is capable of design optimisation with a fully unconstrained core topology.

\section{References}

[1] B. Sobhi-Najafabadi, Optimal Design of Permanent Magnet Generators, PhD Thesis, University of South Australia, Adelaide, Australia, 2002.

[2] Ö. Göl, and J. P. Wieczorek, "Optimal Induction Motor Design by the Use of NLP-Approximation Approach and SQP-Method", Journal of Electric Machines and Power Systems, Vol. 27, No. 6, pp. 602$612,1999$.
[3] W. I. Zangwill, Nonlinear Programming: Sequential A Unified Approach, Prentice-Hall International, Inc., London, U.K, 1969.

[4] M. Huyer, and A. Neumaier, "Global Optimization by Multilevel Coordinate Search", Journal of Global Optimization, Vol. 4, pp. 331-355, 1999.

[5] Ö. Göl and B. Sobhi-Najafabadi, Optimization and Inverse Problems in Electromagnetism, "An Evolutionary Algorithm for the Optimal Design of A Rare Earth Permanent Magnet Machine", (Editors: Marek Rudnicki and Slawomir Wiak), Kluwer Academic Publishers, IBSN 1-4020-1506-2, pp 253260, 2003.

[6] M. A. Branch and A. Grace, MATLAB Optimisation Toolbox The Math Works Inc, 1996.

[7] Ö. Göl and B. Sobhi-Najafabadi, "Permanent Magnet Generator Design: A Systematic Approach", Proceedings of the International Aegean Conference on Electrical Machines and Power Electronics, ACEMP 2001, Kuşadas1, Turkey, 2001, pp. 361-365.

[8] Ö. Göl and B. Sobhi-Najafabadi, "Development of Optimisation Algorithms Applicable to Design of Permanent Magnet Generators", Proceedings of the Second IASTED International Conference, Power and Energy Systems (EuroPES), Crete, Greece, 2002, pp. 484-489. 